\title{
Detection of Salivary Human Papilloma Viruses 16 and 18 (HPV) in Smoker Men in an Iranian Population by PCR: A Pilot Study
}

\author{
Fateme Arbabi-Kalati ${ }^{1}$; Tahereh Nosratzehi ${ }^{2, *}$; Zakaria Bameri ${ }^{3}$; Fiz Mohammad Rigi ${ }^{4}$ \\ ${ }^{1}$ Genetics of Non Communicable Disease Research Center, Department of Oral Medicine, Zahedan University of Medical Sciences \\ ${ }^{2}$ Dental Research center, Department of Oral Medicine, Zahedan University of Medical Sciences, Zahedan, IR Iran \\ 3 Infectious Diseases and Tropical Medicine Research Center, Zahedan University of Medical Sciences, Zahedan, IR Iran \\ ${ }^{4}$ Department of Dentistry, Zahedan University of Medical Sciences, Zahedan, IR Iran \\ ${ }^{*}$ Corresponding author:Tahereh Nosratzehi, Dental Research center, Department of Oral Medicine, Zahedan University of Medical Sciences, Zahedan, IR Iran Tel: +98-5412441814, Fax: \\ +98-5412414003, E-mail: nosratzehi@yahoo.com
}

Received: March 3, 2014; Revised: June 21, 2014; Accepted: June 24, 2014

\begin{abstract}
Background: Human papilloma virus (HPV) infection of the oropharynx is acquired through a variety of sexual and social forms of transmission. Recent epidemiologic evidence has suggested that HPV may be an independent risk factor for oropharyngeal cancers, but risk factors for persistent HPV infection in the oropharynx are unknown. More evidence is needed regarding the prevalence of oral highrisk HPV among healthy smoker and nonsmoker adults.

Objectives: The aim of this study was to compare salivary infection with human papilloma virus types 16 and 18 between smokers and nonsmokers.

Patients and Methods: A hundred healthy adult subjects were selected from Zahedan dental school for this pilot study. DNA was isolated from saliva samples and screened for high-risk HPV strains of HPV16 and 18. Then, further processed using Real Time PCR for quantification and confirmation of sensitivity and specificity of the test. Data was analyzed by t-test.

Results: There were no high-risk types of virus in patients and no significant differences between the groups $(\mathrm{P}=1)$.

Conclusions: It seems that smoking cannot increase the prevalence of high risk HPV16, 18 in saliva samples.

Keywords: DNA Probes, HPV; Saliva; Smoking
\end{abstract}

\section{Background}

Oral cancer is the sixth most common malignancy in the world. Along with major risk factors, like tobacco and alcohol, it is associated with human papilloma virus (HPV) infection and genetic changes. Most of the head and neck cancers are squamous cell carcinomas (SCC). Some studies have suggested that high-risk HPV is a risk factor for SCC and the prevalence of high-risk HPV in head and neck cancers has been reported from $38 \%$ to $100 \%$ in different studies (1). Among all types of HPV, high risk HPV 16 and 18 are the most common viruses in oral cancer (2).

Smoking decreases CD4 lymphocyte and Langerhans cell populations and reduces the activity of natural killer cells; it can also damage the immune system, interfering with HPV infection clearance (3). It is estimated that $50 \%$ of persons with sexual activity become infected with high-risk HPV in their lifetime. Data suggests that oral HPV prevalence is increased with the number of sexual partners and is more typical in men, HIV-infected individuals, and current tobacco users, but their role has not been established (4).

To best our knowledge, no studies are available evaluating the association between smoking and oral high risks HPV infection, but a study showed that oral HPV infection was not found in adults of 20-30 years (5). Evaluation of the prevalence of high-risk HPV in smokers might be useful to understand how smoking induces cancer; it can also be a pilot for a prospective cohort study to compare the incidence of oral SCC in HPV-positive and HPV-negative smokers. If the prevalence of high-risk HPV is higher in smokers, HPV vaccination might be considered in this group. Therefore, the present study was designed to compare salivary contamination with HPV 16 and 18 serotypes between smokers and nonsmokers.

\section{Objectives}

The aim of this study was to compare salivary infection with human papilloma virus types 16 and 18 between smokers and nonsmokers.

\section{Patients and Methods}

This cross-sectional study was performed on 100 adult men. The study protocol was reviewed and approved by the Clinical Ethics Committee of Zahedan University of Medical Sciences, Iran. Patients referring to the department of oral medicine were examined from January to March 2012. 
The exclusion criteria were as follows: history of cervix cancer in the patients' wife, any type of oral lesions, any known systemic diseases, drug use, patients with more than one sexual partner, people without sexual activity, regular use of antimicrobial mouthwash, use of smokeless tobacco, homosexuality and patients with oral sex behavior. Patients who smoke more than five cigarettes in a day, at least for 5 years were considered as smoker. The whole study process was described to patients before informed consent was taken. Patients were divided into two groups: 1) patients without history of smoking and any oral lesion; 2) smoking patients without any oral lesion. The two groups were matched for sex, age, education level, income and social class.

\subsection{Saliva Collection}

At first, patients rinsed their mouth with normal saline for 2 minutes, then the patients were asked to move their tongue over their mouth, after that $5 \mathrm{~mL}$ of unstimulated saliva was collected in a sterile tube with spitting method at oral medicine department. All samples were collected in the morning between 9-11. Saliva samples were transferred in $1.5-\mathrm{mL}$ micro tubes after processing, and then the samples were stored at $-20^{\circ} \mathrm{C}$ until tested.

\subsection{DNA Extraction}

We used pure link viral RNA|DNA kit (Invitrogen company) for DNA extraction (cat No: 12280-050). The protocol of extraction was as follow;

1. We added $25 \mu \mathrm{L}$ proteinase $\mathrm{K}$ into a sterile microcentrifuge

2. We added $200 \mu \mathrm{L}$ sample into microcentrifuge

3. $200 \mu \mathrm{L}$ lysis buffer was added, the tube lid was closed and mixed by vortexing for 15 seconds

4. Incubated at $-56^{\circ} \mathrm{C}$ for 15 minutes

$5.250 \mu \mathrm{L}$ ethanol $100 \%$ was added

6. Incubated at room temperature for 5 minutes

7. Transferred above lysate with ethanol on to viral spin column

8. Centrifuged at $10000 \mathrm{rpm}$ for 1 minute

9. $500 \mu \mathrm{L}$ wash buffer was added

10. Centrifuged at $6800 \mathrm{~g}$ for 1 minute. Discarded the collection tube with flow-through

11. The spin column was placed in a clean wash tube.
Then $500 \mu \mathrm{L}$ wash buffer was added and centrifuged at $6800 \mathrm{~g}$ for 1 minute. The collection tube was discarded with flow-through. The spin column was placed in a clean wash tube

12. Centrifuged at maximum speed for 1 minute. The spin column was placed in a clean wash tube

13.10-50 $\mu \mathrm{L}$ Rnase-free water was added in column

14. Incubated at room temperature for 1 minute

15. The column was centrifuged at the maximum speed for 1 minute

We tested the quality of DNA by electrophoresis and spectrophotometer (ratio 260/280).

\subsection{Real Time PCR}

We tested real time PCR by ABI 7500 instrument. For this step, we used HPV 16 and 18 real time PCR kit with Ref number TD-0030-02 (Liferiver Company). The real time PCR was performed according to the kit protocol. (35 $\mu \mathrm{L}$ Mastermix $+0.4 \mu \mathrm{L}$ enzyme $+1 \mu \mathrm{L}$ internal control $+4 \mu \mathrm{L}$ DNA template, programmed for real time as; $37^{\circ} \mathrm{C}$ for 2 minutes 1 cycle, $94^{\circ} \mathrm{C}$ for 2 minutes 1 cycle, $93^{\circ} \mathrm{C}$ for 15 seconds 40 cycles and $60^{\circ} \mathrm{C}$ for 1 minute 40 cycles). Vic channel were selected for target nucleic acid Fam channel of fluorescence and for internal control. Cycle threshold (CT) value for test was $<35$ and for internal control was 25-33 (Table 1). We used sterile water as a negative control and positive control of the kit. Primer/ probe and positive control were used from special patent of the company.

Data was analyzed using SPSS version 19 software. The independent samples t-test was used.

\section{Results}

A hundred adult men participated in this study (50 patients in the smoking group and 50 patients in nonsmoking group). Their mean ages were $43.1 \pm 9.5$ and 42.4 \pm 10 in the smoking and nonsmoking groups, respectively; there was no significant difference between the two groups $(\mathrm{P}=0.4)$.

Real time PCR results showed that all the patients in the two groups had negative results for HPV 16 and 18 serotypes. Cycle number for internal control was more than 26 , but cycle number for our samples and negative control were less than 35 (Figure 1).

Table 1. Program of Real Time PCR

\begin{tabular}{lc}
\hline & Cycle \\
\hline $37^{\circ} \mathrm{C}$ for 2 minutes & 1 \\
\hline $94^{\circ} \mathrm{C}$ for 2 minutes & 1 \\
\hline $\begin{array}{l}93^{\circ} \mathrm{C} \text { for } 15 \text { seconds, } 60^{\circ} \mathrm{C} \text { for } 1 \text { minute (fluorescence measured at } 60^{\circ} \mathrm{C} \text { ), FAM was target selection } \\
\text { of fluorescence channels and JOE IC }\end{array}$ & 40 \\
\hline
\end{tabular}




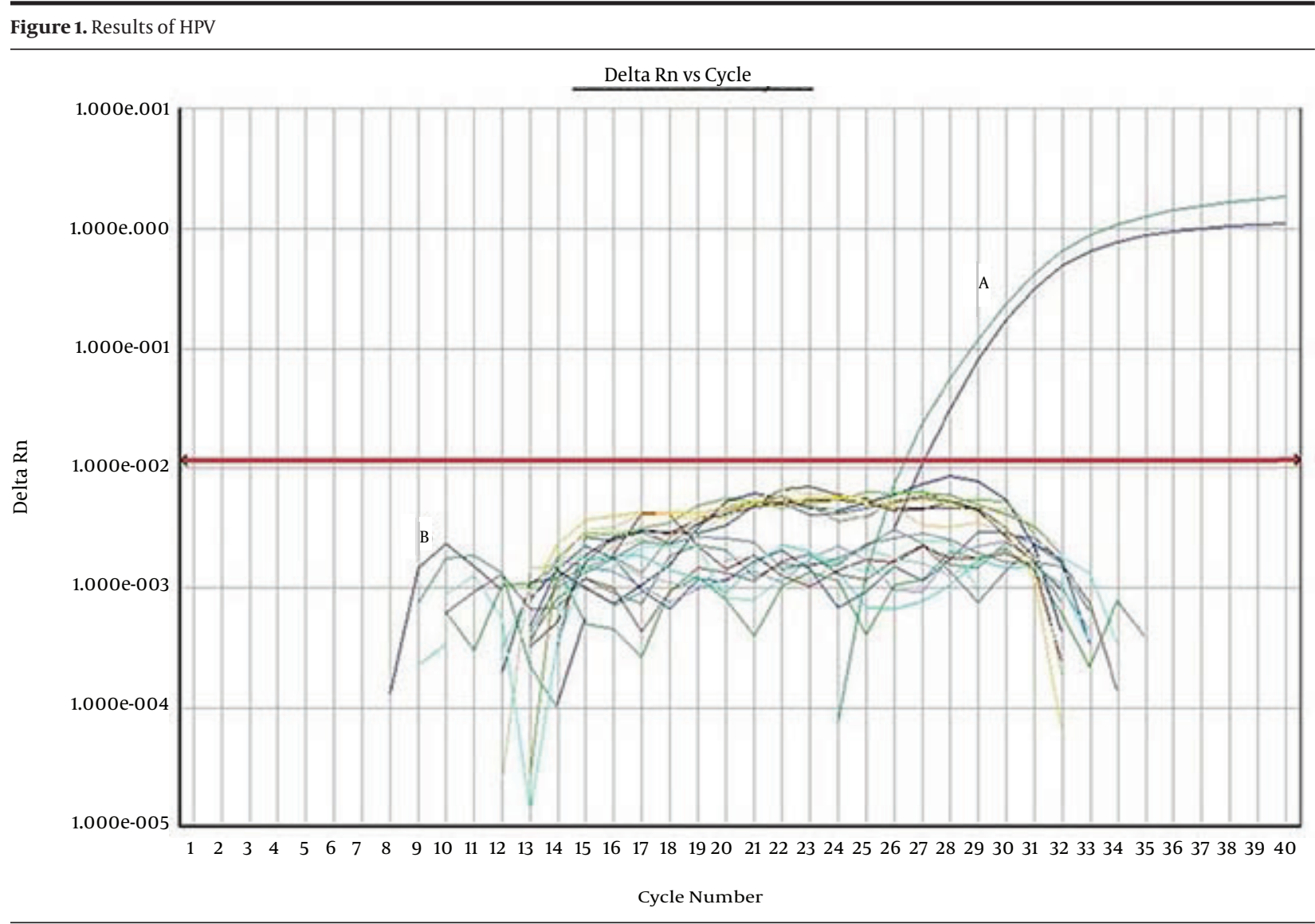

A) Results of HPV 16 and 18 positive controls in PCR kit, cycle number $\geq 26$, B) Results of our samples were tested by real time PCR, cycle number $<35$.

\section{Discussion}

The aim of this study was to evaluate the effect of smoking on salivary contamination with HPV 16 and 18 serotypes. The results showed that smoking did not increase the prevalence of HPV infection and there was no association between HPV infection and patients' education, income and social class.

$\mathrm{HPV}$ is a DNA virus belonging to a large family of viruses, the papovaviridae. HPV infects the skin and mucosa and might induce the formation of both benign and malignant tumors. Oncogenic HPV is well-established as the main risk factor for cervical cancer. HPV infection is now considered as an etiologic agent in SCCs of nasopharynx and base of the tongue. Protein E6 of HPV can cause enzymatic degeneration of p53, which increases cell cycle and impairs DNA repair (6).

No similar studies are available evaluating the effect of smoking on high-risk HPV infection, but several studies have reported HPV infection in healthy individuals with normal oral mucosa. The prevalence of oral and nasopharynx HPV infection is unknown, because sample size, methods of sampling and HPV isolation methods are varied in different studies. Smith et al. assessed 1235 healthy children and teenagers without oral lesions, using exfoliated oral cells. He reported the overall prevalence rates of
$2.5 \%$ in infants, $18 \%$ in children of $1-4$ years, $1.2 \%$ in children of 5-11 years, $1.5 \%$ in teenagers of $12-15$ years and $3.3 \%$ in young adults of 16-20 years for HPV infection. He did not determine HPV types and did not evaluate the possible risk factors; therefore, the results of that study cannot be compared with those of the present study (7).

Montaldo et al. evaluated the prevalence of HPV infection in 146 healthy patients with normal oral mucosa, reporting that salivary HPV 16 serotype was found in $9 \%$ of participants. The results of the present study were different. The effect of smoking or other risk factors were not evaluated; therefore, our results cannot be compared with those of mentioned studies (8).

In another study, Esquenazi et al. reported no HPV detection in 100 healthy individuals, consistent with the results of the present study, but most participants (97\%) in this study were nonsmokers (5). Durzynska et al. evaluated 4149 high school students and reported an HPV prevalence of $1 \%$; however, high-risk HPV was not detected in subjects, which is consistent with the results of the present study. In addition, no association was detected between oral HPV infection and sexual activity in that study (9).

Kreimer et al. evaluated 1680 healthy men in Brazil, Mexico and America and reported the prevalence rates 
of $4 \%$ and 3.1\% for oral HPV infection and high-risk HPV, respectively. It was also reported that smoking could increase the prevalence of oral HPV infection, contrary to the results of the present study. This difference might be attributed to differences in sample sizes (10).

Sanders et al. showed that 3.7\% of Americans had oral HPV, with $1.3 \%$ being oncogenic. In this study, education status, smoking, age, sex and number of sexual partners were not associated with HPV infection (11). Ragin et al. evaluated buccal mucosa cells in 118 healthy women without oral lesions and reported a prevalence of $7.1 \%$ for oral HPV. In that study, prevalence of HPV infection was slightly higher in smokers, which is different from the results of the present study. The difference might be attributed to differences in subjects (women vs. men). However, the prevalence of high-risk HPV was not examined in that study. It was also shown that sexual behavior did not affect the prevalence of HPV (12).

Turner et al. studied 151 healthy adults and reported a prevalence rate of $2.6 \%$ for HPV 16 serotype, which is not consistent with the results of the present study. On the other hand, similar to the present study, none of the subjects had HPV 18 serotype in the oral cavity. The effects of smoking or other risk factors were not examined (2). Gillison et al. evaluated 5579 healthy patients of $14-69$ years and reported prevalence rates of $6.9 \%$ and $1 \%$ for oral HPV infection and HPV 16 serotype, respectively. He reported that smoking can increase the prevalence of oral HPV, which is different from the results of the present study (13).

Gichki et al. investigated the prevalence of oral HPV infection in 192 healthy patients (120 men and 72 women). In his study, $2 \%$ and $5.5 \%$ of patients had positive results for HPV 16 and HPV 18 respectively, also he showed that current smoker combined former smoker had positive results for high risk HPV more than nonsmokers. His results are different with ours, but he did not mention sexual habits of his patients, maybe patients with positive results of this study were homosexual or had several sexual partners. Therefore, we cannot compare the results of these two studies (14).

According to the results of this study, it seems that smoking cannot increase the prevalence of oral HPV 16 and 18 infections. HPV infection is a sexually transmitted disease. Safe sexual activity with a partner could cause negative PCR results in our patients. This study had some limitations: not including women in the study population (we did not want to select just men but due to low smoking habits among women in our society all participants were men in our study between January to March) and small sample size. It is suggested to perform further studies to overcome these limitations and to study other HPV serotypes.

\section{Acknowledgements}

The authors thank the Zahedan University of medical sciences for financial support of this research project.

\section{Authors' Contributions}

Arbabi-Kalati and Rigi drafted the manuscript, managed the literature searches and data analysis; Bameri and Nosratzahi designed the study, guided the data analysis and improved the manuscript. All the authors read and approved the manuscript.

\section{Funding/Support}

This manuscript was supported by Zahedan University of Medical Sciences, Zahedan, IR Iran.

\section{References}

1. Campisi G, Giovannelli L. Controversies surrounding human papilloma virus infection, head \& neck vs oral cancer, implications for prophylaxis and treatment. Head Neck Oncol. 2009;1:8.

2. Turner DO, Williams-Cocks SJ, Bullen R, Catmull J, Falk J, Martin D, et al. High-risk human papillomavirus (HPV) screening and detection in healthy patient saliva samples: a pilot study. BMC Oral Health. 2011;11:28.

3. Vaccarella S, Herrero R, Snijders PJ, Dai M, Thomas JO, Hieu NT, et al. Smoking and human papillomavirus infection: pooled analysis of the International Agency for Research on Cancer HPV Prevalence Surveys. Int J Epidemiol. 2008;37(3):536-46.

4. Marur S, D'Souza G, Westra WH, Forastiere AA. HPV-associated head and neck cancer: a virus-related cancer epidemic. Lancet Oncol. 2010;11(8):781-9.

5. Esquenazi D, Bussoloti Filho I, Carvalho Mda G, Barros FS. The frequency of human papillomavirus findings in normal oral mucosa of healthy people by PCR. Braz J Otorhinolaryngol. 2010;76(1):78-84

6. Feller L, Wood NH, Khammissa RA, Lemmer J. Human papillomavirus-mediated carcinogenesis and HPV-associated oral and oropharyngeal squamous cell carcinoma. Part 2: Human papillomavirus associated oral and oropharyngeal squamous cell carcinoma. Head Face Med. 2010;6:15

7. Smith EM, Swarnavel S, Ritchie JM, Wang D, Haugen TH, Turek LP. Prevalence of human papillomavirus in the oral cavity/oropharynx in a large population of children and adolescents. Pediatr Infect Dis J. 2007;26(9):836-40.

8. Montaldo C, Mastinu A, Quartuccio M, Piras V, Denotti G, Pisano E, et al. Detection and genotyping of human papillomavirus DNA in samples from healthy Sardinian patients: a preliminary study. J Oral Pathol Med. 2007;36(8):482-7.

9. Durzynska J, Pacholska-Bogalska J, Kaczmarek M, Hanc T, Durda M, Skrzypczak M, et al. HPV genotypes in the oral cavity/oropharynx of children and adolescents: cross-sectional survey in Poland. Eur J Pediatr. 2011;170(6):757-61.

10. Kreimer AR, Villa A, Nyitray AG, Abrahamsen M, Papenfuss M, Smith D, et al. The epidemiology of oral HPV infection among a multinational sample of healthy men. Cancer Epidemiol Biomarkers Prev. 2011;20(1):172-82.

11. Sanders AE, Slade GD, Patton LL. National prevalence of oral HPV infection and related risk factors in the US adult population reply. Oral Dis. 2013;19(1):106.

12. Ragin C, Edwards R, Larkins-PM, Taioli E, Eckstein S, Thurman N et al. Oral HPV infection and sexuality: a cross-sectional study in women. Int J Mol Med Sci. 2011;12(6):3928-40.

13. Gillison ML, Broutian T, Pickard RKL, Tong Z, Xiao W, Kahle L, et al Prevalence of oral HPV infection in the United States, 2009-2010. JAMA. 2012;307(7):693-703.

14. Gichki AS, Buajeeb W, Doungudomdacha S, Khovidhunkit SO Detection of human papillomavirus in normal oral cavity in a group of Pakistani subjects using real-time PCR. Asian Pac J Cancer Prev. 2012;13(5):2299-304. 